\title{
On q-ANALOGUE of Differential Subordination Associated with Lemniscate of Bernoulli
}

\author{
Mohsan Raza $\left(\mathbb{D},{ }^{1}\right.$ Hira Naz $\mathbb{D}^{2},{ }^{2}$ Sarfraz Nawaz Malik $\mathbb{D}{ }^{2}$ and Sahidul Islam $\mathbb{D}^{3}$ \\ ${ }^{1}$ Department of Mathematics, Government College University Faisalabad, Faisalabad 38000, Pakistan \\ ${ }^{2}$ Department of Mathematics, COMSATS University Islamabad, Wah Campus, Wah Cantt 47040, Pakistan \\ ${ }^{3}$ Department of Mathematics, Jahangirnagar University, Savar, Dhaka, Bangladesh
}

Correspondence should be addressed to Sahidul Islam; sahidul.sohag@juniv.edu

Received 21 September 2021; Accepted 15 October 2021; Published 1 November 2021

Academic Editor: Fairouz Tchier

Copyright (c) 2021 Mohsan Raza et al. This is an open access article distributed under the Creative Commons Attribution License, which permits unrestricted use, distribution, and reproduction in any medium, provided the original work is properly cited.

This article comprises the study of differential subordination with analogue of $q$-derivative. It includes the sufficient condition on $\gamma$ for $1+\left(\gamma \partial z_{q} h(z) / h^{n}(z)\right)$ to be subordinated by $(1+A z / 1+B z),-1 \leq B<A \leq 1$, and implies that $h(z)<\sqrt{1+z}$, where $h(z)$ is the analytic function in the open unit disk. Moreover, certain sufficient conditions for $q$-starlikeness of analytic functions related with lemniscate of Bernoulli are determined.

\section{Introduction}

Let a set $\mathscr{A}$ be considered as the class of analytic functions defined in open unit disk $\mathbb{U}=\{\varsigma: \varsigma \in \mathbb{C}$ and $|\varsigma|<1\}$ under normalization conditions $f(0)=0$ and $f^{\prime}(0)=1$, having

$$
f(\varsigma)=\varsigma+\sum_{n=2}^{\infty} a_{n} \varsigma^{n}, \quad \varsigma \in \mathbb{U}
$$

as Taylor series. The class $S$ comprises the normalized univalent functions, defined in $\mathbb{U}$. The major subcategories of class $S$ are $C$ of convex functions and $S^{*}$ of starlike functions. The class $P$ is another important class of analytic univalent functions whose co-domains are restricted to the right half plane and are used to determine the convexity and starlikeness of univalent functions. For more details, see $[1,2]$.

Let $f$ and $g$ be two analytic functions in $\mathbb{U}$. Then, $f$ is subordinated by $g$, denoted as $f<g$ if $f$ can be written in the form of composition of $g$ and $₫$ as $f(\varsigma)=g(\varpi(\varsigma))$ subject to the existence of analytic function $₫$ which satisfies the condition that $\Phi(0)=0$ and $|\varpi(\varsigma)|<|\varsigma|$. Furthermore, if both $f$ and $g$ are univalent functions in $\mathbb{U}$, then $f<g$ implies that $f(0)=g(0)$ and $f(\mathbb{U}) \subset g(\mathbb{U})$.

Subordination plays an important role in univalent function theory, and this concept was first introduced by Lindelöf, but Littlewood $[3,4]$ contributed remarkably to this field.
Differential subordination is actually the generalized version of differential inequalities with real variables. Many researchers contributed in the work related to differential subordinations. Historical developments in the field of differential subordination are briefly described by Miller and Mocanu in [5].

The advancement in the field of differential subordination starts with the usage of univalent functions. It was noticed in an article by Miller et al. [6] in 1974. Furthermore, many developments in this field have been achieved with the usage of differential subordinations in past fifty years. Differential inequality was a very well-known concept of real variables, and to study it in terms of complex variables, Miller and Mocanu [7] in 1981 were the first ones to introduce the idea of differential subordination. The contribution of Ruscheweyh and Singh [8] and Ruscheweyh and Wilken [9] is also of great importance in this field. Wellknown Jack's lemma [10] has brought the advancements in differential subordinations. Dziok [11] worked on some of the applications of Jack's lemma. The research work carried out by Ma and Minda [12] in the function theory is worth to mention here, as they introduced the analytic function $\Phi$, which satisfies the conditions of normalization $\Phi(0)=0$ and $\Phi^{\prime}(0)>1$ having real part positive. The authors in [12] utilized the function $\Phi$ and introduced the subclass $\mathcal{S}^{*}(\Phi)$ of starlike functions as follows: 


$$
\mathcal{S}^{*}(\Phi)=\left\{f \in \mathscr{A}: \frac{\varsigma f^{\prime}(\varsigma)}{f(\varsigma)} \prec \Phi(\varsigma) ; \quad \varsigma \in \mathbb{U}\right\} .
$$

The idea presented in [12] is very useful, and it helped many researchers for further studies in this direction. Ali et al. $[13,14]$ worked on differential subordination for sufficiency criteria of Janowski starlikeness and evaluated several differential subordinations such as $1+\gamma \varsigma$ $\left(p^{\prime}(\varsigma) / p^{n}(\varsigma)\right)$ and found $p(\varsigma) \prec \sqrt{1+\varsigma}$. Also, Ravichandran et al. [15] used this concept to find the sufficient conditions for starlikeness of Bernoulli's lemniscate and Janowski functions. Sharma et al. [16] studied the differential subordinations to prove the starlikeness associated with cardioid domain and Halim et al. [17] introduced the concept for limacon domain.

Jackson $[18,19]$ was the one who introduced the $q$-derivatives and $q$-integrals. After Jackson, Srivastava was amongst the pioneers to contribute in the $q$-calculus for its usage for analytic functions and their subclasses. Not only this, but he also applied $q$-hypergeometric function in the functions theory. All these contributions are comprised in his book (pp. 347 in [20]). Ismail et al. [21] contributed in the $q$-calculus for the study of starlike functions. Anastassiu and Gal $[22,23]$ also played their part in the development of complex variables with $q$-generalization. Purohit et al. [24] have used fractional $q$-calculus operators to apply subordination conditions on the class of non-Bazilevic functions. Sahoo and Agrawal [25] worked on starlike functions in $q$-calculus and extended the idea of $q$-starlikeness for particular subclasses of starlike functions. The involvement of $q$-derivative in the class $\mathcal{S}^{*}(\Phi)$ gave the formation of following subclass $\mathcal{S}_{q}^{*}(\Phi)$ of starlike functions which was introduced by Aouf and Seoudy [26].

$$
\mathcal{S}_{q}^{*}(\Phi)=\left\{f \in \mathscr{A}: \frac{\varsigma D_{q} f(\varsigma)}{f(\varsigma)}<\Phi(\varsigma) ; \quad \varsigma \in \mathbb{U}\right\} .
$$

The class described above has drawn the attention of many researchers. Replacing $\Phi(\varsigma)$ with different functions such as Janowski, lemniscate of Bernoulli, cardioid, and limacon, the researchers got the new directions to the study. Srivastava et al. [27] studied $q$-derivatives to find the relation between different classes of $q$-starlike functions related to Janowski function. Srivastava et al. [28] introduced the class of $q$-starlike functions by using general conic domains. They also obtained the bounds on Hankel and Toeplitz determinants for $q$-starlike functions and continued working par excellence. They produced unmatchable results that worked as great motivation for many researchers worldwide. To have an idea of their remarkable work, one can see [29-31], [20, 27, 28, 32-43]. Contributions of Haq et al. [44] and Zainab et al. [45] are also worth to mention. They studied $q$-analogue of differential subordinations for star-like functions related to limacon and cardioid domains, and Janowski functions. The $q$-derivative is the foundation of all this work in $q$-analogue, and it is defined as follows.

The $q$-derivative of a complex valued function $f$, defined in the domain $\mathbb{U}$, is given as follows:

$$
\left(D_{q} f\right)(\varsigma)= \begin{cases}\frac{f(\varsigma)-f(q \varsigma)}{(1-q) \varsigma}, & \varsigma \neq 0, \\ f^{\prime}(0), & \varsigma=0,\end{cases}
$$

where $0<q<1$. This implies the following:

$$
\lim _{q \longrightarrow 1^{-}}\left(D_{q} f\right)(\varsigma)=\lim _{q \longrightarrow 1^{-}} \frac{f(\varsigma)-f(q \varsigma)}{(1-q) \varsigma}=f^{\prime}(\varsigma),
$$

on the assumption that the function $f$ is differentiable in $\mathbb{U}$. The $q$-derivative $D_{q} f$ of an analytic function $f$ has Taylor series of the form

$$
\left(D_{q} f\right)(\varsigma)=\sum_{n=0}^{\infty}[n]_{q} a_{n} \varsigma^{n-1}
$$

where

$$
[n]_{q}= \begin{cases}\frac{1-q^{n}}{1-q}, & n \in \mathbb{C}, \\ \sum_{k=0}^{n-1} q^{k}, & n \in \mathbb{N} .\end{cases}
$$

For more details about $q$-derivative and recent work on it, we refer the readers to [29-31], [20, 27, 28, 32-43]. In addition, the $q$-analogue of Jack's lemma has played a vital role in this paper which states as follows.

Lemma 1 (see [46]). Let $₫$ be an analytic function in $\mathbb{U}$ with $\omega(0)=0$. For maximum of $\Phi$ on $|\varsigma|=1$ at $\varsigma_{0}=a e^{i \theta}$, where $\theta \in[-\pi, \pi]$ and $0<q<1$, then we have

$$
\varsigma_{0} D_{q} \bowtie\left(\varsigma_{0}\right)=m \bowtie\left(\varsigma_{0}\right),
$$

where $m \in \mathbb{R}$ with $m \geq 1$.

\section{Main Results}

Theorem 1. Assume that

$$
|\gamma| \geq \frac{(A-B)(\sqrt{2}+\sqrt{3-q})}{(1-|B|)}, \quad-1<B<A \leq 1 .
$$

Consider an analytic function $h$ on $\mathbb{U}$ with $h(0)=1$ which satisfies

$$
1+\gamma \varsigma D_{q} h(\varsigma) \prec \frac{1+A \varsigma}{1+B \varsigma}, \quad \varsigma \in \mathbb{U} .
$$

Also, suppose

$$
1+\gamma \varsigma D_{q} h(\varsigma)=\frac{1+A \omega(\varsigma)}{1+B \omega(\varsigma)}, \quad \varsigma \in \mathbb{U} .
$$

Here, $\Phi$ is an analytic function in $\mathbb{U}$ such that $\Phi(0)=0$. Then, we have

$$
h(\varsigma) \prec \sqrt{1+\varsigma} \text {. }
$$

Proof. Suppose that 


$$
p(\varsigma)=1+\gamma \varsigma D_{q} h(\varsigma)
$$

where $p$ is analytic, and we have $p(0)=1$. Also, consider that

$$
h(\varsigma)=\sqrt{1+\Phi(\varsigma)}
$$

Now, we prove that $|\oplus(\varsigma)|<1$, where

$$
\omega(\varsigma)=\frac{p(\varsigma)-1}{A-B p(\varsigma)} \text {. }
$$

Using (13) and (14), we obtain

$$
p(\varsigma)=1+\gamma \varsigma \frac{D_{q} \omega(\varsigma)}{\sqrt{1+\omega(\varsigma)}+\sqrt{1+\omega(\varsigma)-\varsigma D_{q} \omega(\varsigma)(1-q)}} .
$$

Also, we have

$$
\left|\frac{p(\varsigma)-1}{A-B p(\varsigma)}\right|=\left|\frac{\gamma \varsigma D_{q} \omega(\varsigma)}{(A-B)\left[\sqrt{1+\Phi(\varsigma)}+\sqrt{1+\varpi(\varsigma)-\varsigma D_{q} \varpi(\varsigma)(1-q)}\right]-B \gamma \varsigma D_{q} \omega(\varsigma)}\right| .
$$

Consider a point $\varsigma_{0} \in \mathbb{U}$ such that

$$
\max _{|\varsigma| \leq\left|\varsigma_{0}\right|}|\oplus(\varsigma)|=\left|\oplus\left(\varsigma_{0}\right)\right|=1 \text {. }
$$

Now, by using Lemma 1, we have $\varsigma_{0} D_{q} \omega\left(\varsigma_{0}\right)=m \omega\left(\varsigma_{0}\right), \quad m \geq 1$. Now, consider that $\Phi\left(\varsigma_{0}\right)=e^{i \theta}, \quad \theta \in[-\pi, \pi]$; then, for $\varsigma_{0} \in \mathbb{U}$, we obtain

$$
\begin{aligned}
\left|\frac{p\left(\varsigma_{0}\right)-1}{A-B p\left(\varsigma_{0}\right)}\right| & =\left|\frac{\gamma \varsigma_{0} D_{q} \Phi\left(\varsigma_{0}\right)}{(A-B)\left[\sqrt{1+\Phi\left(\varsigma_{0}\right)}+\sqrt{1+\Phi\left(\varsigma_{0}\right)-\varsigma_{0} D_{q} \Phi\left(\varsigma_{0}\right)(1-q)}\right]-B \gamma \varsigma_{0} D_{q} \Phi\left(\varsigma_{0}\right)}\right| \\
& \geq \frac{|\gamma| m}{(A-B)\left[\sqrt{|1|+\mid e^{i \theta \mid}}+\sqrt{|1|+\left|e^{i \theta}\right|+\left|m e^{i \theta}(1-q)\right|}\right]+|B||\gamma| m}, \\
& =\frac{|\gamma| m}{(A-B)[\sqrt{2}+\sqrt{2+m(1-q)}]+|B||\gamma| m} .
\end{aligned}
$$

Consider a new function

Then,

$$
\Xi(m)=\frac{|\gamma| m}{(A-B)[\sqrt{2}+\sqrt{2+m(1-q)}]+|B||\gamma| m} .
$$

$$
\Xi^{\prime}(m)=\frac{|\gamma|[(A-B)\{\sqrt{2}+\sqrt{2+m(1-q)}\}]-|\gamma| m[((A-B)(1-q) / 2 \sqrt{2+m(1-q)})]}{[(A-B)\{\sqrt{2}+\sqrt{2+m(1-q)}\}+|B||\gamma| m]^{2}}>0 .
$$

Above expression represents that the function $\Xi$ has increasing behavior, so we have its minimum value at $m=1$ and

$$
\Xi(1)=\frac{|\gamma|}{(A-B)[\sqrt{2}+\sqrt{3-q}]+|B||\gamma|} .
$$

So, we conclude that

$$
\left|\frac{p\left(\varsigma_{0}\right)-1}{A-B p\left(\varsigma_{0}\right)}\right| \geq \frac{|\gamma|}{(A-B)[\sqrt{2}+\sqrt{3-q}]+|B||\gamma|} \text {. }
$$

Now, from (9), we have

$$
\left|\frac{p\left(\varsigma_{0}\right)-1}{A-B p\left(\varsigma_{0}\right)}\right| \geq 1,
$$

Since this result contradicts (10), therefore, $|\bowtie(\varsigma)|<1$, which completes the proof.

By taking $h(\varsigma)=\left(\varsigma D_{q} f(\varsigma) / f(\varsigma)\right)$, we deduce the following result.

Corollary 1. Let $|\gamma| \geq((A-B)(\sqrt{2}+\sqrt{3-q}) /(1-|B|))$, $-1<B<A \leq 1$ and $f \in \mathscr{A}$, satisfy the subordination 


$$
1+\gamma \varsigma D_{q}\left(\frac{\varsigma D_{q} f(\varsigma)}{f(\varsigma)}\right) \prec \frac{1+A \varsigma}{1+B \varsigma}
$$

Then, $f \in \mathcal{S}_{q}^{*}(\sqrt{1+\varsigma})$.

Theorem 2. Assume that

$$
|\gamma| \geq \frac{\sqrt{2}(A-B)(\sqrt{2}+\sqrt{3-q})}{(1-|B|)}, \quad-1<B<A \leq 1 .
$$

Consider an analytic function $h$ on $\mathbb{U}$ with $h(0)=1$ which satisfies

$$
1+\frac{\gamma \varsigma D_{q} h(\varsigma)}{h(\varsigma)} \prec \frac{1+A \varsigma}{1+B \varsigma}, \quad \varsigma \in \mathbb{U} .
$$

Also, suppose

$$
1+\frac{\gamma \varsigma D_{q} h(\varsigma)}{h(\varsigma)}=\frac{1+A \varpi(\varsigma)}{1+B \oplus(\varsigma)}, \quad \varsigma \in \mathbb{U}
$$

where $\Phi$ is analytic function on $\mathbb{U}$ with $\Phi(0)=0$. Then,

$$
h(\varsigma) \prec \sqrt{1+\varsigma} \text {. }
$$

Proof. We define a function

$$
p(\varsigma)=1+\frac{\gamma \varsigma D_{q} h(\varsigma)}{h(\varsigma)}
$$

where $p$ is analytic and $p(0)=1$. Now, consider that

$$
h(\varsigma)=\sqrt{1+\Phi(\varsigma)}
$$

To obtain the result, we have to show that $|\bowtie(\varsigma)|<1$. Using (30) and (31), we obtain the result

$$
p(\varsigma)=1+\gamma \varsigma \frac{D_{q} \omega(\varsigma)}{\sqrt{1+\Phi(\varsigma)}\left[\sqrt{1+\Phi(\varsigma)}+\sqrt{1+\Phi(\varsigma)-\varsigma D_{q} \Phi(\varsigma)(1-q)}\right]}
$$

Also, we have

$$
\left|\frac{p(\varsigma)-1}{A-B p(\varsigma)}\right|=\left|\frac{\gamma \varsigma D_{q} \omega(\varsigma)}{(A-B) \sqrt{1+\omega(\varsigma)}\left[\sqrt{1+\omega(\varsigma)}+\sqrt{1+\omega(\varsigma)-\varsigma D_{q} \omega(\varsigma)(1-q)}\right]-B \gamma \varsigma D_{q} \omega(\varsigma)}\right| .
$$

Consider a point $\varsigma_{0} \in \mathbb{U}$ such that

$$
\max _{|\varsigma| \leq\left|\varsigma_{0}\right|}|\omega(\varsigma)|=\left|\oplus\left(\varsigma_{0}\right)\right|=1
$$

Now, by using Lemma 1, we have $\varsigma_{0} D_{q} \Phi\left(\varsigma_{0}\right)=m \omega\left(\varsigma_{0}\right), \quad m \geq 1$. Now, consider that $\omega\left(\varsigma_{0}\right)=e^{i \theta}, \quad \theta \in[-\pi, \pi]$; then, for $\varsigma_{0} \in \mathbb{U}$, we obtain

$$
\begin{aligned}
\left|\frac{p\left(\varsigma_{0}\right)-1}{A-B p\left(\varsigma_{0}\right)}\right| & =\left|\frac{\gamma m e^{i \theta}}{(A-B) \sqrt{1+e^{i \theta}}\left[\sqrt{1+e^{i \theta}}+\sqrt{1+e^{i \theta}-m e^{i \theta}(1-q)}\right]-B \gamma m e^{i \theta}}\right|, \\
& \geq \frac{|\gamma| m}{(A-B) \sqrt{|1|+\left|e^{i \theta \mid}\right|}\left[\sqrt{|1|+\left|e^{i \theta}\right|}+\sqrt{|1|+\left|e^{i \theta}\right|+\left|m e^{i \theta}(1-q)\right|}\right]+|B||\gamma| m}, \\
& =\frac{|\gamma| m}{(A-B) \sqrt{2}[\sqrt{2}+\sqrt{2+m(1-q)}]+|B||\gamma| m} .
\end{aligned}
$$

\section{Consider}




$$
\Xi_{1}(m)=\frac{|\gamma| m}{(A-B) \sqrt{2}[\sqrt{2}+\sqrt{2+m(1-q)}]+|B||\gamma| m} .
$$

Then,

$$
\Xi_{1}^{\prime}(m)=\frac{|\gamma|[(A-B) \sqrt{2}\{\sqrt{2}+\sqrt{2+m(1-q)}\}]-|\gamma| m[(\sqrt{2}(A-B)(1-q) / 2 \sqrt{2+m(1-q)})]}{[(A-B) \sqrt{2}\{\sqrt{2}+\sqrt{2+m(1-q)}\}+|B||\gamma| m]^{2}}>0 .
$$

Above expression represents that function $\Xi_{1}$ has increasing behavior, so we have its minimum value at $m=1$ and

$$
\Xi_{1}(1)=\frac{|\gamma|}{(A-B) \sqrt{2}[\sqrt{2}+\sqrt{3-q}]+|B||\gamma|} .
$$

So, we conclude that

$$
\left|\frac{p\left(\varsigma_{0}\right)-1}{A-B p\left(\varsigma_{0}\right)}\right| \geq \frac{|\gamma|}{(A-B) \sqrt{2}[\sqrt{2}+\sqrt{3-q}]+|B||\gamma|} \text {. }
$$

Now, from (26), we have

$$
\left|\frac{p\left(\varsigma_{0}\right)-1}{A-B p\left(\varsigma_{0}\right)}\right| \geq 1
$$

which contradicts (27), and hence, $|\bowtie(\varsigma)|<1$, which completes the proof.

By taking $h(\varsigma)=\left(\varsigma D_{q} f(\varsigma) / f(\varsigma)\right)$, we deduce the following result.

Corollary 2. Let $\quad|\gamma| \geq \sqrt{2}((A-B)(\sqrt{2}+\sqrt{3-q}) /$ $(1-|B|)), \quad-1<B<A \leq 1$ and $f \in \mathscr{A}$, which satisfies the subordination

$$
1+\gamma \varsigma\left(\frac{f(\varsigma)}{\varsigma D_{q} f(\varsigma)}\right) D_{q}\left(\frac{\varsigma D_{q} f(\varsigma)}{f(\varsigma)}\right) \prec \frac{1+A \varsigma}{1+B \varsigma}
$$

Then, $f \in \mathcal{S}_{q}^{*}(\sqrt{1+\varsigma})$.

$$
|\gamma| \geq \frac{2(A-B)(\sqrt{2}+\sqrt{3-q})}{(1-|B|)}, \quad-1<B<A \leq 1 .
$$

Consider an analytic function $h$ on $\mathbb{U}$ with $h(0)=1$ which satisfies

$$
1+\frac{\gamma \varsigma D_{q} h(\varsigma)}{h^{2}(\varsigma)} \prec \frac{1+A \varsigma}{1+B \varsigma}, \quad \varsigma \in \mathbb{U} .
$$

Also, suppose

$$
1+\frac{\gamma \varsigma D_{q} h(\varsigma)}{h^{2}(\varsigma)}=\frac{1+A \omega(\varsigma)}{1+B \varpi(\varsigma)}, \quad \varsigma \in \mathbb{U},
$$

where $₫$ is analytic function on $\mathbb{U}$ with $₫(0)=0$. Then,

$$
h(\varsigma) \prec \sqrt{1+\varsigma} .
$$

Proof. We define a function

$$
p(\varsigma)=1+\frac{\gamma \varsigma D_{q} h(\varsigma)}{h^{2}(\varsigma)}
$$

where $p$ is analytic, and we have $p(0)=1$. Now, consider that

$$
h(\varsigma)=\sqrt{1+\omega(\varsigma)} .
$$

To obtain the result, we have to show that $|\bowtie(\varsigma)|<1$. Using (46) and (47), we obtain the result

Theorem 3. Assume that

$$
p(\varsigma)=1+\gamma \varsigma \frac{D_{q} \Phi(\varsigma)}{\sqrt{1+\omega(\varsigma)}\left[\sqrt{1+\omega(\varsigma)}+\sqrt{1+\omega(\varsigma)-\varsigma D_{q} \Phi(\varsigma)(1-q)}\right]}
$$

Also, we have

$$
\left|\frac{p(\varsigma)-1}{A-B p(\varsigma)}\right|=\left|\frac{\gamma \varsigma D_{q} \omega(\varsigma)}{(A-B) \sqrt{1+\Phi(\varsigma)}\left[\sqrt{1+\omega(\varsigma)}+\sqrt{1+\Phi(\varsigma)-\varsigma D_{q} @(\varsigma)(1-q)}\right]-B \gamma \varsigma D_{q} \omega(\varsigma)}\right| .
$$


Consider a point $\varsigma_{0} \in \mathbb{U}$ such that

$$
\max _{|\varsigma| \leq\left|\varsigma_{0}\right|}|\omega(\varsigma)|=\left|\omega\left(\varsigma_{0}\right)\right|=1 \text {. }
$$

Now, by using Lemma 1, we have $\varsigma_{0} D_{q} \Phi\left(\varsigma_{0}\right)=m \omega\left(\varsigma_{0}\right), \quad m \geq 1$. Now, consider that $\varpi\left(\varsigma_{0}\right)=e^{i \theta}, \quad \theta \in[-\pi, \pi]$; then, for $\varsigma_{0} \in \mathbb{U}$, we obtain

$$
\begin{aligned}
\left|\frac{p\left(\varsigma_{0}\right)-1}{A-B p\left(\varsigma_{0}\right)}\right| & =\left|\frac{\gamma m e^{i \theta}}{(A-B) \sqrt{1+e^{i \theta}}\left[\sqrt{1+e^{i \theta}}+\sqrt{1+e^{i \theta}-m e^{i \theta}(1-q)}\right]-B \gamma m e^{i \theta}}\right| \\
& \geq \frac{|\gamma| m}{(A-B) \sqrt{|1|+\left|e^{i \theta}\right|}\left[\sqrt{|1|+\left|e^{i \theta}\right|}+\sqrt{|1|+\left|e^{i \theta}\right|+\left|m e^{i \theta}(1-q)\right|}\right]+|B||\gamma| m}, \\
& =\frac{|\gamma| m}{(A-B) 2[\sqrt{2}+\sqrt{2+m(1-q)}]+|B||\gamma| m} .
\end{aligned}
$$

Consider a function

Then,

$$
\Xi_{2}(m)=\frac{|\gamma| m}{(A-B) 2[\sqrt{2}+\sqrt{2+m(1-q)}]+|B||\gamma| m} .
$$

$$
\Xi_{2}^{\prime}(m)=\frac{|\gamma|[(A-B) 2\{\sqrt{2}+\sqrt{2+m(1-q)}\}]-|\gamma| m[(2(A-B)(1-q) / 2 \sqrt{2+m(1-q)})]}{[(A-B) 2\{\sqrt{2}+\sqrt{2+m(1-q)}\}+|B||\gamma| m]^{2}}>0 .
$$

Here, $\Xi_{2}$ is clearly an increasing function, so we have its minimum value at $m=1$ and

$$
\Xi_{2}(1)=\frac{|\gamma|}{2(A-B)[\sqrt{2}+\sqrt{3-q}]+|B||\gamma|}
$$

So, we conclude that

$$
\left|\frac{p\left(\varsigma_{0}\right)-1}{A-B p\left(\varsigma_{0}\right)}\right| \geq \frac{|\gamma|}{2(A-B)[\sqrt{2}+\sqrt{3-q}]+|B||\gamma|} .
$$

Now, from (42), we have

$$
\left|\frac{p\left(\varsigma_{0}\right)-1}{A-B p\left(\varsigma_{0}\right)}\right| \geq 1
$$

which contradict (43), and hence, $|\bowtie(\varsigma)|<1$, which completes the proof.

By taking $h(\varsigma)=\left(\varsigma D_{q} f(\varsigma) / f(\varsigma)\right)$, we deduce the following result.

Corollary 3. Let $|\gamma| \geq 2((A-B)(\sqrt{2}+\sqrt{3-q}) /(1-|B|))$, $-1<B<A \leq 1$ and $f \in \mathscr{A}$, satisfies the subordination

$$
1+\gamma \varsigma\left(\frac{f(\varsigma)}{\varsigma D_{q} f(\varsigma)}\right)^{2} D_{q}\left(\frac{\varsigma D_{q} f(\varsigma)}{f(\varsigma)}\right) \prec \frac{1+A \varsigma}{1+B \varsigma} .
$$

Then, $f \in \mathcal{S}_{q}^{*}(\sqrt{1+\varsigma})$.

Theorem 4. Assume that

$$
|\gamma| \geq \frac{2 \sqrt{2}(A-B)(\sqrt{2}+\sqrt{3-q})}{(1-|B|)}, \quad-1<B<A \leq 1 .
$$

Consider an analytic function $h$ on $\mathbb{U}$ with $h(0)=1$ which satisfies

$$
1+\frac{\gamma \varsigma D_{q} h(\varsigma)}{h^{3}(\varsigma)} \prec \frac{1+A \varsigma}{1+B \varsigma}, \quad \varsigma \in \mathbb{U} .
$$

Also, suppose

$$
1+\frac{\gamma \varsigma D_{q} h(\varsigma)}{h^{3}(\varsigma)}=\frac{1+A \omega(\varsigma)}{1+B \omega(\varsigma)}, \quad \varsigma \in \mathbb{U},
$$

where $₫$ is analytic function on $\mathbb{U}$ with $₫(0)=0$. Then,

$$
h(\varsigma) \prec \sqrt{1+\varsigma} \text {. }
$$

Proof. We define a function

$$
p(\varsigma)=1+\frac{\gamma \varsigma D_{q} h(\varsigma)}{h^{3}(\varsigma)}
$$


where $p$ is analytic, and we have $p(0)=1$. Now, consider that

$$
h(\varsigma)=\sqrt{1+\Phi(\varsigma)}
$$

To obtain the result, we have to show that $|ळ(\varsigma)|<1$. Using (62) and (63), we obtain

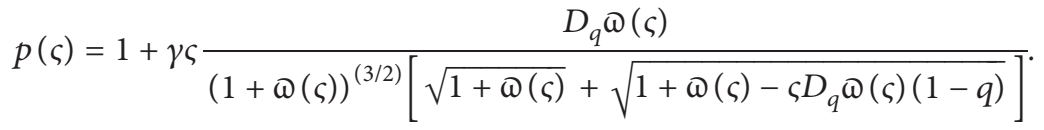

Also, we have

$$
\left|\frac{p(\varsigma)-1}{A-B p(\varsigma)}\right|=\left|\frac{\gamma \varsigma D_{q} \omega(\varsigma)}{(A-B)(1+\omega(\varsigma))^{(3 / 2)}\left[\sqrt{1+\omega(\varsigma)}+\sqrt{1+\omega(\varsigma)-\varsigma D_{q} \omega(\varsigma)(1-q)}\right]-B \gamma \varsigma D_{q} \omega(\varsigma)}\right| .
$$

Consider a point $\varsigma_{0} \in \mathbb{U}$ such that

$$
\max _{|\varsigma| \leq\left|\varsigma_{0}\right|}|\oplus(\varsigma)|=\left|\oplus\left(\varsigma_{0}\right)\right|=1 .
$$

Now, by using Lemma 1, we have $\varsigma_{0} D_{q} \omega\left(\varsigma_{0}\right)=m \omega\left(\varsigma_{0}\right), \quad m \geq 1$. Now, consider that $\Phi\left(\varsigma_{0}\right)=e^{i \theta}, \quad \theta \in[-\pi, \pi]$; then, for $\varsigma_{0} \in \mathbb{U}$, we obtain

$$
\begin{aligned}
\left|\frac{p\left(\varsigma_{0}\right)-1}{A-B p\left(\varsigma_{0}\right)}\right| & =\mid \frac{\gamma m e^{i \theta}}{(A-B)\left(1+e^{i \theta}\right)^{(3 / 2)}\left[\sqrt{1+e^{i \theta}}+\sqrt{1+e^{i \theta}-m e^{i \theta}(1-q)}\right]-B \gamma m e^{i \theta} \mid}, \\
& \geq \frac{|\gamma| m}{(A-B)\left(|1|+\left|e^{i \theta}\right|\right)^{(3 / 2)}\left[\sqrt{|1|+\left|e^{i \theta}\right|}+\sqrt{|1|+\left|e^{i \theta}\right|+\left|m e^{i \theta}(1-q)\right|}\right]+|B||\gamma| m}, \\
& =\frac{|\gamma| m}{(A-B) 2^{(3 / 2)}[\sqrt{2}+\sqrt{2+m(1-q)}]+|B||\gamma| m} .
\end{aligned}
$$

Consider a function

$\Xi_{3}(m)=\frac{|\gamma| m}{2^{(3 / 2)}(A-B)[\sqrt{2}+\sqrt{2+m(1-q)}]+|B||\gamma| m}$.
Then,

$$
\Xi_{3}^{\prime}(m)=\frac{|\gamma|\left[(A-B) 2^{(3 / 2)}\{\sqrt{2}+\sqrt{2+m(1-q)}\}\right]-|\gamma| m\left[\left(2^{(3 / 2)}(A-B)(1-q) / 2 \sqrt{2+m(1-q)}\right)\right]}{\left[(A-B) 2^{(3 / 2)}\{\sqrt{2}+\sqrt{2+m(1-q)}\}+|B||\gamma| m\right]^{2}}>0 .
$$


Above expression represents that function $\Xi_{3}$ has increasing behavior, so we have its minimum value at $m=1$ and

$$
\Xi_{3}(1)=\frac{|\gamma|}{2^{(3 / 2)}(A-B)[\sqrt{2}+\sqrt{3-q}]+|B||\gamma|} .
$$

So, we conclude that

$$
\left|\frac{p\left(\varsigma_{0}\right)-1}{A-B p\left(\varsigma_{0}\right)}\right| \geq \frac{|\gamma|}{2^{(3 / 2)}(A-B)[\sqrt{2}+\sqrt{3-q}]+|B||\gamma|} .
$$

Now, from (58), we have

$$
\left|\frac{p\left(\varsigma_{0}\right)-1}{A-B p\left(\varsigma_{0}\right)}\right| \geq 1,
$$

which contradicts (59), and hence, $|\varpi(\varsigma)|<1$, which completes the proof.

By taking $h(\varsigma)=\left(\varsigma D_{q} f(\varsigma) / f(\varsigma)\right)$, we deduce the following result.

Corollary 5. Let $|\gamma| \geq 2 \sqrt{2}((A-B)(\sqrt{2}+\sqrt{3-q}) /$ $(1-|B|)),-1<B<A \leq 1$ and $f \in \mathscr{A}$, satisfy the subordination

$$
1+\gamma \varsigma\left(\frac{f(\varsigma)}{\varsigma D_{q} f(\varsigma)}\right)^{3} D_{q}\left(\frac{\varsigma D_{q} f(\varsigma)}{f(\varsigma)}\right)<\frac{1+A \varsigma}{1+B \varsigma} .
$$

Then, $f \in \mathcal{S}_{q}^{*}(\sqrt{1+\varsigma})$.

Theorem 6. Assume that

$$
|\gamma| \geq \frac{2^{(n / 2)}(A-B)(\sqrt{2}+\sqrt{3-q})}{(1-|B|)}, \quad-1<B<A \leq 1 .
$$

Consider an analytic function $h$ on $\mathbb{U}$ with $h(0)=1$ which satisfies

$$
1+\frac{\gamma \varsigma D_{q} h(\varsigma)}{h^{n}(\varsigma)}<\frac{1+A \varsigma}{1+B \varsigma}, \quad \varsigma \in \mathbb{U} .
$$

Also, suppose

$$
1+\frac{\gamma \varsigma D_{q} h(\varsigma)}{h^{n}(\varsigma)}=\frac{1+A \varpi(\varsigma)}{1+B \varpi(\varsigma)}, \quad \varsigma \in \mathbb{U},
$$

where $₫$ is analytic function on $\mathbb{U}$ with $₫(0)=0$. Then,

$$
h(\varsigma) \prec \sqrt{1+\varsigma} \text {. }
$$

Proof. We define a function

$$
p(\varsigma)=1+\frac{\gamma \varsigma D_{q} h(\varsigma)}{h^{n}(\varsigma)}
$$

where $p$ is analytic, and we have $p(0)=1$. Now, consider that

$$
h(\varsigma)=\sqrt{1+\Phi(\varsigma)}
$$

To obtain the result, we have to show that $|\bowtie(\varsigma)|<1$. Using (78) and (79), we obtain

$$
p(\varsigma)=1+\gamma \varsigma \frac{D_{q} \varpi(\varsigma)}{(1+\omega(\varsigma))^{(n / 2)}\left[\sqrt{1+\omega(\varsigma)}+\sqrt{1+\omega(\varsigma)-\varsigma D_{q} \omega(\varsigma)(1-q)}\right]} .
$$

Also, we have

$$
\begin{aligned}
& \left|\frac{p(\varsigma)-1}{A-B p(\varsigma)}\right|=\left|\frac{\gamma \varsigma\left(D_{q} \omega(\varsigma) /(1+\omega(\varsigma))^{(n / 2)}\left[\sqrt{1+\omega(\varsigma)}+\sqrt{1+\Phi(\varsigma)-\varsigma D_{q} \Phi(\varsigma)(1-q)}\right]\right)}{A-B\left[1+\gamma \varsigma\left(D_{q} \Phi(\varsigma) /(1+\Phi(\varsigma))^{(n / 2)}\left[\sqrt{1+\Phi(\varsigma)}+\sqrt{1+\Phi(\varsigma)-\varsigma D_{q} \Phi(\varsigma)(1-q)}\right]\right)\right]}\right| \\
& =\left|\frac{\gamma \varsigma D_{q} \omega(\varsigma)}{(A-B)(1+\Phi(\varsigma))^{(n / 2)}\left[\sqrt{1+\Phi(\varsigma)}+\sqrt{1+\Phi(\varsigma)-\varsigma D_{q} \omega(\varsigma)(1-q)}\right]-B \gamma \varsigma D_{q} \Phi(\varsigma)}\right| .
\end{aligned}
$$

Consider a point $\varsigma_{0} \in \mathbb{U}$ such that

$$
\max _{|\varsigma| \leq\left|\varsigma_{0}\right|}|\bowtie(\varsigma)|=\left|\bowtie\left(\varsigma_{0}\right)\right|=1 \text {. }
$$

Now, by using Lemma 1, we have $\varsigma_{0} D_{q} \omega\left(\varsigma_{0}\right)=m \varpi\left(\varsigma_{0}\right), \quad m \geq 1$. Now, consider that $Ф\left(\varsigma_{0}\right)=e^{i \theta}, \quad \theta \in[-\pi, \pi]$; then, for $\varsigma_{0} \in \mathbb{U}$, we obtain 


$$
\begin{aligned}
& \left|\frac{p\left(\varsigma_{0}\right)-1}{A-B p\left(\varsigma_{0}\right)}\right|=\frac{\gamma \varsigma_{0} D_{q} \varpi\left(\varsigma_{0}\right)}{(A-B)\left(1+\varpi\left(\varsigma_{0}\right)\right)^{(n / 2)}\left[\sqrt{1+\varpi\left(\varsigma_{0}\right)}+\sqrt{1+\varpi\left(\varsigma_{0}\right)-\varsigma_{0} D_{q} \Phi\left(\varsigma_{0}\right)(1-q)}\right]-B \gamma \varsigma_{0} D_{q} \varpi\left(\varsigma_{0}\right)}, \\
& =\left|\frac{\gamma m e^{i \theta}}{(A-B)\left(1+e^{i \theta}\right)^{(n / 2)}\left[\sqrt{1+e^{i \theta}}+\sqrt{1+e^{i \theta}-m e^{i \theta}(1-q)}\right]-B \gamma m e^{i \theta}}\right| \text {, } \\
& \geq \frac{|\gamma| m}{(A-B)\left(1+e^{i \theta}\right)^{(n / 2)}\left[\left|\sqrt{1+e^{i \theta}}\right|+\left|\sqrt{1+e^{i \theta}-m e^{i \theta}(1-q)}\right|\right]-|B||\gamma| m}, \\
& =\frac{|\gamma| m}{(A-B)\left(|1|+\left|e^{i \theta}\right|\right)^{(n / 2)}\left[\sqrt{\left|1+e^{i \theta}\right|}+\sqrt{\left|1+e^{i \theta}-m e^{i \theta}(1-q)\right|}\right]+|B||\gamma| m}, \\
& \geq \frac{|\gamma| m}{(A-B)\left(|1|+\left|e^{i \theta}\right|\right)^{(n / 2)}\left[\sqrt{|1|+\left|e^{i \theta}\right|}+\sqrt{|1|+\left|e^{i \theta}\right|+\left|m e^{i \theta}(1-q)\right|}\right]+|B||\gamma| m}, \\
& =\frac{|\gamma| m}{(A-B) 2^{(n / 2)}[\sqrt{2}+\sqrt{2+m(1-q)}]+|B||\gamma| m} .
\end{aligned}
$$

Consider

Then,

$\Xi_{4}(m)=\frac{|\gamma| m}{2^{(n / 2)}(A-B)[\sqrt{2}+\sqrt{2+m(1-q)}]+|B||\gamma| m}$.

$$
\Xi_{4}^{\prime}(m)=\frac{|\gamma|\left[(A-B) 2^{(n / 2)}\{\sqrt{2}+\sqrt{2+m(1-q)}\}\right]-|\gamma| m\left[\left(2^{(n / 2)}(A-B)(1-q) / 2 \sqrt{2+m(1-q)}\right)\right]}{\left[(A-B) 2^{(n / 2)}\{\sqrt{2}+\sqrt{2+m(1-q)}\}+|B||\gamma| m\right]^{2}}>0
$$

Above expression represents that function $\Xi_{4}$ has increasing behavior, so we have its minimum value at $m=1$ and

$$
\Xi_{4}(1)=\frac{|\gamma|}{2^{(n / 2)}(A-B)[\sqrt{2}+\sqrt{3-q}]+|B||\gamma|} .
$$

So, we conclude that

$$
\left|\frac{p\left(\varsigma_{0}\right)-1}{A-B p\left(\varsigma_{0}\right)}\right| \geq \frac{|\gamma|}{2^{(n / 2)}(A-B)[\sqrt{2}+\sqrt{3-q}]+|B||\gamma|} .
$$

Now, from (74), we have

$$
\left|\frac{p\left(\varsigma_{0}\right)-1}{A-B p\left(\varsigma_{0}\right)}\right| \geq 1
$$

which contradict (75), and hence, $|\varpi(\varsigma)|<1$, which completes the proof.

By taking $h(\varsigma)=\left(\varsigma D_{q} f(\varsigma) / f(\varsigma)\right)$, we deduce the following result.

Corollary 7. Let $|\gamma| \geq\left(2^{(n / 2)}(A-B)(\sqrt{2}+\sqrt{3-q}) /\right.$ $(1-|B|)), \quad-1<B<A \leq 1$ and $f \in \mathscr{A}$, satisfy the subordination

$$
1+\gamma \varsigma\left(\frac{f(\varsigma)}{\varsigma D_{q} f(\varsigma)}\right)^{n} D_{q}\left(\frac{\varsigma D_{q} f(\varsigma)}{f(\varsigma)}\right) \prec \frac{1+A \varsigma}{1+B \varsigma} .
$$

Then, $f \in \mathcal{S}_{q}^{*}(\sqrt{1+\varsigma})$. 


\section{Conclusion}

In this article, we have investigated the $q$-differential subordination by using $q$-version of well-known Jack's Lemma. We have found the condition on $\gamma$ such that $1+\left(\gamma \varsigma D_{q} h(\varsigma) / h^{n}(\varsigma)\right) \prec(1+A \varsigma / 1+B \varsigma) \quad$ implies that $h(\varsigma) \prec \sqrt{1+\varsigma}$. These results have been utilized to find sufficient conditions for star-like functions related to lemniscate of Bernoulli. This method can further be applied to find sufficient conditions for star-like functions of Ma-Minda type.

\section{Data Availability}

No data were used in this article.

\section{Conflicts of Interest}

The authors declare that they have no conflicts of interest.

\section{Authors' Contributions}

All authors contributed equally and approved the final manuscript.

\section{References}

[1] P. L. Duren, Univalent functions. Grundlehren der Math. Wissenchaften, Springer-Verlag, New York, NY, USA, 1983.

[2] A. W. Goodman, "Univalent functions," Mariner Publishing Company, vol. 1, no. 2, 1983.

[3] J. E. Littlewood, Lectures on the Theory of Functions, Oxford University Press, London, UK, 1944.

[4] J. E. Littlewood, "On inequalities in the theory of functions," Proceedings of the London Mathematical Society, vol. 2, no. 23, pp. 481-519, 2002.

[5] S. S. Miller and P. T. Mocanu, Differential Subordinations Theory and Applications, Marcel Dekker, New York, NY, USA, 2000.

[6] S. S. Miller, P. T. Mocanu, and M. O. Reade, "Bazilevic functions and generalized convexity," Revue Roumaine de Mathématique Pures et Appliquées, vol. 19, pp. 213-224, 1974.

[7] S. S. Miller and P. T. Mocanu, "Differential subordinations and univalent functions," Michigan Mathematical Journal, vol. 28, pp. 157-171, 1981.

[8] S. Ruscheweyh and V. Singh, "On a Briot-Bouquet equation related to univalent functions," Revue Roumaine de Mathématique Pures et Appliquées, vol. 24, pp. 285-290, 1979.

[9] S. Ruscheweyh and D. R. Wilken, "Sharp estimates for certain Briot-Bouquet subordinations," Revue Roumaine de Mathématique Pures et Appliquées, vol. 30, pp. 559-569, 1985.

[10] I. S. Jack, "Functions starlike and convex of order $\alpha$," Journal of the London Mathematical Society, vol. 2-3, no. 3, pp. 469-474, 1971.

[11] D. Jacek, "Applications of the Jack lemma," Acta Mathematica Hungaria, vol. 105, pp. 93-102, 2004.

[12] W. C. Ma and D. A. Minda, "Unified treatment of some special classes of univalent functions," in Proceedings of the Conference on Complex Analysis, pp. 157-169, International Press, Cambridge, MA, USA, 1992.

[13] R. M. Ali, V. Ravichndaran, and N. Seenivasagan, "Sufficient conditions for janoswski starlikeness," International Journal of Mathematics and Mathematical Sciences, vol. 23, no. 9, p. 56, 2007.

[14] R. M. Ali, N. E. Cho, V. Ravichandran, and S. S. Kumar, "Differential subordination for functions associated with the lemniscate of Bernoulli. Taiwan," Jurnal Matematika, vol. 16, pp. 1017-1026, 2012.

[15] K. Sivaprasad, K. Virendra, V. Ravichandran, and E. C. Nak, "Sufficient conditions for starlike functions associated with the lemniscates of Bernoulli," Journal of Inequalities and Applications, vol. 173, 2013.

[16] K. Sharma, N. K. Jain, and V. Ravichandran, "Starlike functions associated with a cardioid," Afrika Matematika, vol. 27, no. 5-6, pp. 923-939, 2016.

[17] Y. Yunus, S. A. Halim, and A. B. Akbarally, "Subclass of starlike functions associated with a limacon," AIP Conference Proceedings, vol. 1974, Article ID 030023, 2018.

[18] F. H. Jackson, "On $q$-functions and certain difference operator," Transactions of the Royal Society of Edinburgh, vol. 46, pp. 253-281, 1908.

[19] F. H. Jackson, "On $q$-definite integrals," Quarterly Journal of Pure and Applied Mathematics, vol. 41, pp. 193-203, 1910.

[20] H. M. Srivastava, "Univalent functions, fractional calculus, and associated generalized hypergeometric functions in Univalent Functions," in Fractional Calculus, and Their Applications, H. M. Srivastava and S. Owa, Eds., pp. 329-354, Wileya, J. and Sons, New York, NY, USA, 1989.

[21] M. E. Ismail, E. Merkes, and D. Styer, "A generalization of Starlike functions," Theory and Applications, vol. 14, no. 4, pp. 77-84, 1990.

[22] G. A. Anastassiu and S. G. Gal, "Geometric and approximation properties of some singular integrals in the unit disk," Journal of Inequalities and Applications, vol. 1, p. 19, Article ID 17231, 2006.

[23] G. A. Anastassiu and S. G. Gal, "Geometric and approximation properties of generalized singular integrals," Journal of the Korean Mathematical Society, vol. 23, no. 2, pp. 425-443, 2006.

[24] S. Abelman, K. A. Selvakumaran, M. M. Rashidi, and S. D. Purohit, "Subordination conditions for a class of nonbazilevič type defined by using fractional Q-calculus operators," Facta Universitatis - Series: Mathematics and Informatics, vol. 32, no. 2, pp. 255-267, 2017.

[25] S. Agrawal and S. K. Sahoo, "A generalization of starlike functions of order alpha," Hokkaido Mathematical Journal, vol. 46, pp. 15-27, 2017.

[26] T. M. Seoudy and M. K. Aouf, "Coefficient estimates of new classes of $q$-starlike and $q$-convex functions of complex order," Journal of Mathematical Inequalities, vol. 10, no. 1, pp. 135-145, 2016.

[27] H. M. Srivastava, M. Tahir, B. Khan, Q. Z. Ahmad, and N. Khan, "Some general classes of $q$-starlike functions associated with the Janowski functions," Symmetry Plus, vol. 11, Article ID 292, 2019.

[28] H. M. Srivastava, M. Tahir, B. Khan, Q. Ahmad, and N. Khan, "Some general families of $q$-starlike functions associated with the Janowski functions," Filomat, vol. 33, no. 9, pp. 2613-2626, 2019.

[29] B. Khan, H. M. Srivastava, N. Khan, M. Darus, M. Tahir, and Q. Z. Ahmad, "Coefficient estimates for a subclass of analytic functions associated with a certain leaf-like domain," Mathematics, vol. 8, no. 8, p. 1334, 2020.

[30] S. Mahmood, M. Jabeen, S. N. Malik, H. M. Srivastava, R. Manzoor, and S. M. J. Riaz, "Some coefficient inequalities of $q$-starlike functions associated with conic domain defined by 
q-derivative," Journal of Function Spaces, vol. 2018, Article ID 8492072, 13 pages, 2018.

[31] M. Shafiq, H. M. Srivastava, N. Khan, Q. Z. Ahmad, M. Darus, and S. Kiran, "An upper bound of the third Hankel determinant for a subclass of $q$-starlike functions associated with k-fibonacci numbers," Symmetry Plus, vol. 12, no. 6, p. 1043, 2020.

[32] H. M. Srivastava, "Operators of basic (or $q$-) calculus and fractional $q$-calculus and their applications in geometric function theory of complex analysis," Iranian Journal of Science and Technology Transaction A-Science, vol. 44, no. 1, pp. 327-344, 2020

[33] H. M. Srivastava, Q. Z. Ahmad, N. Khan, N. Khan, and B. Khan, "Hankel and Toeplitz determinants for a subclass of $q$-starlike functions associated with a general conic domain," Mathematics, vol. 7, Article ID 181, 2019.

[34] H. M. Srivastava, M. K. Aouf, and A. O. Mostafa, "Some properties of analytic functions associated with fractional $q$-calculus operators q-calculus operators," Miskolc Mathematical Notes, vol. 20, no. 2, pp. 1245-1260, 2019.

[35] H. M. Srivastava, M. Arif, M. Arif, and M. Raza, "Convolution properties of meromorphically harmonic functions defined by a generalized convolution $\$ q$-derivative operator," AIMS Mathematics, vol. 6, no. 6, pp. 5869-5885, 2021.

[36] H. M. Srivastava, S. Arjika, and A. S. Kelil, "Some homogeneous $q$-difference operators and the associated generalized Hahn polynomials," Applied Set-Valued Analysis and Optimization, vol. 1, pp. 187-201, 2019.

[37] H. M. Srivastava and D. Bansal, "Close-to-convexity of a certain family of q-Mittag-Leffler functions," Journal of Nonlinear and Variational Analysis, vol. 1, pp. 61-69, 2017.

[38] H. M. Srivastava, J. Cao, and S. Arjika, "A note on generalized $q$-difference equations and their applications involving q-hypergeometric functions," Symmetry Plus, vol. 12, Article ID 1816, 2020.

[39] H. M. Srivastava and S. Arjika, "A general family of $q$-hypergeometric polynomials and associated generating functions," Mathematics, vol. 9, Article ID 1161, 2021.

[40] H. M. Srivastava, B. Khan, N. Khan, and Q. Z. Ahmad, "Coefficient inequalities for $q$-starlike functions associated with the Janowski functions," Hokkaido Mathematical Journal, vol. 48, pp. 407-425, 2019.

[41] H. M. Srivastava, B. Khan, N. Khan, M. Tahir, S. Ahmad, and N. Khan, "Upper bound of the third Hankel determinant for a subclass of $q$-starlike functions associated with the $q$-exponential function," Bulletin des Sciences Mathematiques, vol. 167, Article ID 102942, 2021.

[42] H. M. Srivastava, S. Khan, S. Khan, Q. Z. Ahmad, N. Khan, and S. Hussain, "The Faber polynomial expansion method and its application to the general coefficient problem for some subclasses of bi-univalent functions associated with a certain $q$-integral operator," Studia Universitatis Babes-Bolyai Matematica, vol. 63, no. 4, pp. 419-436, 2018.

[43] H. M. Srivastava, N. Raza, E. S. A. AbuJarad, G. Srivastava, and M. H. AbuJarad, "Fekete-Szegö inequality for classes of $(p, q)$-Starlike and $(p, q)$-convex functions," Revista de la Real Academia de Ciencias Exactas, Físicas y Naturales. Serie A. Matemáticas, vol. 113, no. 4, pp. 3563-3584, 2019.

[44] M. Ul-Haq, M. Raza, M. Arif, Q. Khan, and H. Tang, "Qanalogue of differential subordinations," Mathematics, vol. 7, no. 8, p. 724, 2019.
[45] S. Zainab, A. Shakeel, M. Imran et al., "Sufficiency criteria for q-starlike functions associated with cardioid," Journal of Function Spaces, vol. 2021, Article ID 9999213, 9 pages, 2021.

[46] A. Çetinkaya and Y. Polatoglu, " $q$-Harmonic mappings for which analytic part is q-convex functions of complex order," Hacettepe Journal of Mathematics and Statistics, vol. 47, pp. 813-820, 2018. 